\title{
A calibration cover for multi-spectral space instruments
}

Nicola Baccichet, Roberta Aló, Stephan Gulde, Dominik Magner, Mika Tajiri

Nicola Baccichet, Roberta Aló, Stephan Gulde, Dominik Magner, Mika Tajiri, "A calibration cover for multi-spectral space instruments," Proc. SPIE 11852, International Conference on Space Optics - ICSO 2020, 118526H (11 June 2021); doi: 10.1117/12.2600298

SPIE Event: International Conference on Space Optics - ICSO 2021, 2021, Online Only 


\section{International Conference on Space Optics-ICSO 2020}

Virtual Conference

30 March-2 April 2021

Edited by Bruno Cugny, Zoran Sodnik, and Nikos Karafolas
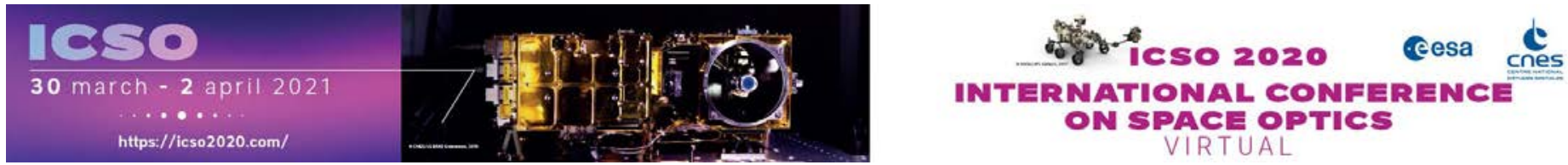

\section{A calibration cover for multi-spectral space instruments}

\section{Cesa isopmeatians ecnes}

International Conference on Space Optics - ICSO 2020, edited by Bruno Cugny, Zoran Sodnik, Nikos Karafolas, Proc. of SPIE Vol. 11852, 118526H · ( 2021 ESA and CNES

CCC code: $0277-786 \mathrm{X} / 21 / \$ 21 \cdot$ doi: $10.1117 / 12.2600298$ 


\title{
A calibration cover for multi-spectral space instruments
}

\author{
Nicola Baccichet*, Roberta Aló, Stephan Gulde, Dominik Magner \\ OHB Systems AG, Manfred-Fuchs-Strasse 1, 82234 Wessling, Germany
}

\begin{abstract}
The study of many astrophysical objects relies on repeated measurements of specific areas of the sky to allow, for example, the identification of transient phenomena, or of targets of interest extracted automatically from a large set of observations. A key aspect coming to play in these cases, is the stability of the measurement system that allows data to be traceable and comparable over time, which in turn leads to the necessity of a reliable and stable calibration.

Observations from space allows for an intrinsically more stable environment due to the absence of ground-generated disturbances, leading to a less complex calibration system to correct for effects such as ageing of optics/detectors or temperature variations in the instrument. In this context, making use of external sources reduces further the need for internal devices, minimizing the impact of calibration in the design of a spacecraft.

In this paper we present the performance of a device that takes advantage of these aspects and makes use of the sun to passively generate calibration scenes at visible and infrared wavelengths, with a single part. It consists on a movable cover placed at the entrance pupil of a telescope, with pinhole inserts and a black-coated internal surface. Sunlight passing through the pinholes is used to generate a flat field at visible wavelengths, while by heating the inner surface an infrared uniform scene can be formed.
\end{abstract}

We apply this concept to a case-study for a small-sized satellite (e.g. 6U CubeSat) showing a good relative stability with such system in both IR and VIS bands, over the course of a 2-3 years mission.

Keywords: CubeSat, radiometric calibration, mid-IR, visible, cover

\section{INTRODUCTION}

The modern optical instruments used in astrophysics to gather high-resolution data, provided either by large ground telescopes or state-of-the-art space observatories, generally make use of low-resolution observations to prioritize the analyses of interesting targets. Especially when studying transient phenomena that rely on a continuous observation of the sky, these become a fundamental tool to compensate the limited observing time availability to single users offered by advanced facilities such as the VLT or the upcoming ELTs and JWST. In addition, low-resolutions automated surveys offers a way to quickly and regularly observe specific parts of the sky (or its entirety) to increase the detection of variable objects or a faster way to follow-up on objects of interests in the realm of multi-messenger astronomy.

In this context, observations from space using CubeSats offer a higher flexibility and efficiency compared to small telescopes on ground, since they are not bound by atmospheric conditions or the day/night cycles. Due to their standardized components and use in the EO domain, sensors and compact optical systems are becoming increasingly available, potentially allowing astronomers to build small satellite platforms using mostly off-the-shelf components that reduces the development time as well as the overall launch and hardware costs.

The viability of employing small satellites for transient monitoring or general astronomical observations is proven by the several missions that being proposed and also concepts already being validated with flying prototypes (for example: [1] [3]). Specifically in the context of photometric-based astrophysics, a small space observatory would provide valuable information for many science cases, for example: microlensing, monitoring of the galactic center, exoplanets transits, variable object detection or search for asteroids and comets, or supernovae. Additionally, a desirable feature in this scenario, is the capability of observing simultaneously in two different wavelength ranges, as it allows enhanced detection capabilities and possibilities to automatically categorize the images and determine the most interesting follow-up targets.

*nicola.baccichet@ohb.de 
A key aspect for these kind of observations is being able to reliably trace over time the photometric response of the system, consequently requiring a stable and repeatable calibration strategy - preferably automated to guarantee compatibility with the handling of a continuous flow of data and automated image processing algorithms. When relying for absolute calibration on stellar standards, a faster relative radiometric calibration can be efficiently sufficient to map the short and mid-term changes in the optical instrument under use. Generally, in this case built-in systems inside the spacecraft are used, but this solution is of limited viability in the case of CubeSats - opening the option for use of external devices instead. It is in this context that, taking advantage of OHB's professional expertise in developing state-of-the art optical payloads, we analysed a solution for an external calibration device to be used in such applications - owing to the design and analyses capabilities developed for the study of large optical payloads such as those on board PLATO, MTG, or the upcoming CHIME, and FORUM study.

Specifically, we analysed the possibility of employing a moveable cover with calibration functions to be used in a $6 \mathrm{U}$ CubeSat with both visible and mid-IR broadband observation capabilities. Apart from its active mechanism, the sources are passively created by taking advantage of the sun to provide calibration sources in both channels. When closed, small pinholes allow to directly observe the sun with the visible detector and build a flat-field by scanning its image across the focal plane, while in the infrared its black-painted surface can be heat-up to provide a sufficiently bright source to illuminate the mid-IR focal plane.

In this paper we use the concept of a CubeSat housing two detectors to illustrate the potential performance of such a calibration device. Firstly we introduce the system under study and its main calibration functions (section 2), then in section 3 we analyse the key feasibility aspects such as its thermal behavior and the required calibration signal and finally in section 4 give an overview of the main expected performance in terms of relative radiometric stability.

\section{DESIGN CONCEPT}

\subsection{CubeSat concept}

To analyse the concept mentioned above, we consider a $6 \mathrm{U}$ CubeSat inside which $2.5 \mathrm{U}$ are allocated to the optical relay, $1 \mathrm{U}$ to the dichroic splitting the visible and infrared channels and the two cameras, and the remaining to electronics, ADCS and motors. To collect the stellar radiation we consider a compact 3-mirrors optical relay, similar to those proposed already in a number of CubeSat design ([4] - [8]) providing roughly an f/6 system with a FoV of 0.16 degrees - key specifications in Table 1.

The detection system consists in two cameras, a broadband visible CMOS (400-900 nm) and a mid-IR microbolometer operating in the thermal infrared (around the $8-15 \mu \mathrm{m}$ range). Also in this case, we take reference data from detector systems available in the CubeSat market to define the specific detector parameter basing on available designs (e.g. [4]) and off-theshelf items. Particularly, we consider a panchromatic camera in the visible range similar to that specified in [1] as well as an uncooled microbolometer with performance similar to [9] [10] - summary of key parameters in Table 2.

The CubeSat is foreseen to be launched on a drifting low-Earth orbit, at 700km height. In this configuration, the satellite automatically acquires images in the two channels, in short exposures to allow for frequent mapping of specific portions of the sky - in the search for bright transient events. At this height, the satellite speed would be around $7.5 \mathrm{~km} / \mathrm{s}$, with an orbital period of around 100min, providing 14.6 revolutions per day. We consider a mission lifetime of 3 years and assume that the CubeSat attitude is controlled via three reaction wheels delivering and absolute pointing accuracy of $0.1^{\circ}$.

Key to the monitoring activity required to detect transient events, is the radiometric stability of the system, which is achieved by regularly calibrating both channels, with background and flat-field acquisitions. As introduced in the section before, the calibration takes advantage of a moveable aluminium cover mounted on the outer surface of the CubeSat, one side of which is covered in black coating (e.g. Aeroglaze) and the other side protected with MLI, with four pinholes mounted on it. Conceptually, the calibration sequence consist on a two-point (gain/offset) calibration, done by acquiring regularly background and bright flat-field images. It is assumed that the on-board system is used only for relative radiometric calibration and monitoring of effects such as ageing of the optics and detector degradation, since a more precise absolute calibration can be carried out observing stellar standards. 


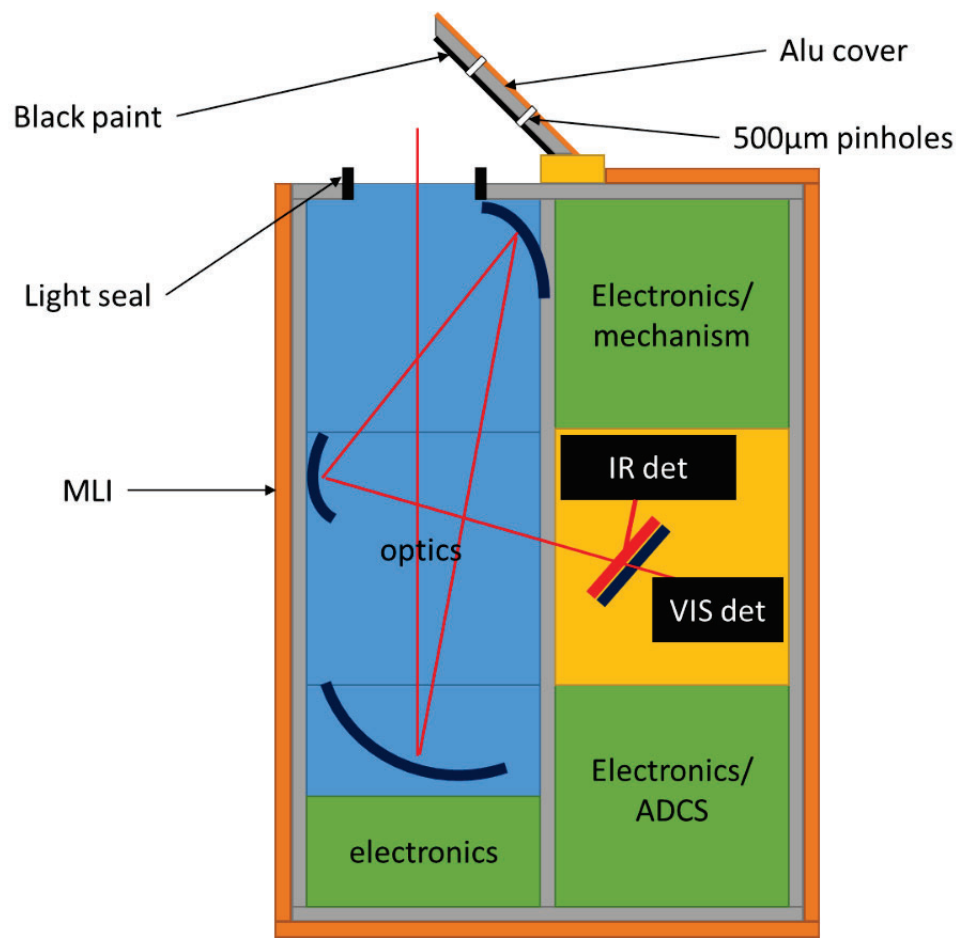

Figure 1: conceptual representation of the $6 \mathrm{U}$ CubeSat design under study. The colours indicate the different function of each unit, blue for CubeSat units housing the optical system, light orange for the detector and beam splitting unit and green for the units housing control electronics for the mechanisms, mass memory, batteries, communication and ADCS (attitude determination and control system). The orange lines surrounding the CubeSat represent a thin MLI substrate that covers most of its outer shell; not represented are the deployable solar panels assumed to be mounted on all the four long sides of the CubeSat.

Table 1: main optical parameters of the CubeSat design under consideration.

\begin{tabular}{|l|l|l|}
\hline Parameter & VIS & Mid-IR \\
\hline f-number & 6.25 & 6.25 \\
\hline Aperture & $8 \mathrm{~cm}$ & $8 \mathrm{~cm}$ \\
\hline Field of view & $0.16^{\circ}$ & $0.16^{\circ}$ \\
\hline Mirrors reflectivity & 0.95 & 0.9 \\
\hline
\end{tabular}

Table 2: main parameters of the detection system under consideration.

\begin{tabular}{|l|l|l|}
\hline Parameter & VIS & Mid-IR \\
\hline Detector size & $1 \mathrm{k} \times 1 \mathrm{k}$ & $640 \mathrm{x} 480$ \\
\hline Pixel pitch & $5 \mu \mathrm{m}$ & $15 \mu \mathrm{m}$ \\
\hline Pixel scale & $2.1 \mathrm{arcsec} / \mathrm{px}$ & $6.2 \mathrm{arcsec} / \mathrm{px}$ \\
\hline Wavelength range & $400-900 \mathrm{~nm}$ & $8-14 \mu \mathrm{m}$ \\
\hline QE & $60 \%$ & - \\
\hline
\end{tabular}




\begin{tabular}{|l|l|l|}
\hline Detector well & 15000 & - \\
\hline Detector noise & $50 \mathrm{e}-$ & - \\
\hline NEDT & - & $50 \mathrm{mK}$ at $60 \mathrm{~Hz}$ \\
\hline
\end{tabular}

Table 3: principal cover and calibration parameters considered in the study.

\begin{tabular}{|l|l|}
\hline Parameter & Value \\
\hline Cover active diameter & $8 \mathrm{~cm}$ \\
\hline Coating emissivity & 0.9 (VIS) - 0.95 (Mid-IR) \\
\hline Number of pinholes & 4 \\
\hline Pinholes diameter & $500 \mu \mathrm{m}$ \\
\hline $\begin{array}{l}\text { Exposure time } \\
\text { (observations and calibration) }\end{array}$ & $0.15 \mathrm{~ms}$ (VIS) / 3ms (IR) \\
\hline
\end{tabular}

\subsection{Calibration concept}

Since a relatively good stability of the detectors is foreseen (which is also well characterizable on-ground), a gain calibration is assumed to be sufficient every 7 days - period after which the orbit has sufficiently drifted and the thermal environment's changes becomes significant (especially in IR).

The sequence foreseen begins by calibrating the mid-IR channel (Figure 2), they key part of which consist in pointing the black side of the half-open cover to the sun for about 5-10 minutes to heat it up to about $370 \mathrm{~K}$. This is then closed to allow flat-fields to be measured. Temperature sensors mounted on the cover allows to compensate the difference in radiance emitted by the cover due to the change of the incident solar radiation throughout the year. In this scenario, we consider that background acquisitions are done before and after the flat-field ones and subsequently weighted to determine the calibration background. This is done through a series of chop/nod acquisitions centered around a relatively dark part of the sky (which will vary depending on the position of the cubesat around the orbit). We assume that in this case the image take is carried out in a short amount of time, therefore no considerable variation in the temperature of the optics is expected (generally the timescale in which optics and the structure respond to temperature changes is longer compared to the calibration timescale).

After that, to calibrate the visible channel the closed cover is still used, this time by observing the sun through its pinholes (Figure 3). In this case, the flat-field is created by averaging over various frames acquired while scanning over the sun. To allow sufficient signal every calibration frame, a relatively slow scan speed is considered (around $0.6 \%$ ). With this speed, scanning the sun disc over the focal plane while acquiring images at the exposure time of Table 3 , leads to a total calibration time of about 1.2 seconds, assuming 10 frames averaging every 20 pixels. Subsequently, dark images are acquired again by pointing with the cover closed to a relatively dark part of the sky, since we can assume that the radiance transmitted through the pinholes in this configuration is negligible. Similarly to the IR case, also when performing the sun calibration it is assumed that the radiance entering the system is scaled during post-processing for the Earth-Sun distance variation over the year.

In addition to the calibration sequences described above, since IR observations are strongly affected by the instrument temperatures, a regular daily calibration is considered as well. Every orbit, at different positions around it, a few chop/nod background acquisitions allow minimizing the difference between the calibration background and the IR scene, by following the temperature variations of the satellite while minimizing the impact on the time available for science acquisitions. Assuming a 3ms exposure and a target of acquiring 100 frames (chop/nod differences) per day, this leads to a reserved calibration time of less than $0.002 \%$ (of a day).

Moreover, one can take advantage of the larger detector area compared to the active FoV (i.e. exposed to external radiation) to further improve the accuracy of background subtraction in both channels. In the visible, unexposed pixels allow 
compensating for the temperature-dependent level of the detector dark, using the calibrated background to compensate for non-uniformities in the response of each individual pixel. In the infrared, monitoring the drift of the detector's surrounding structure allows the scaling of the background level generated by the optics in-between the calibration events.

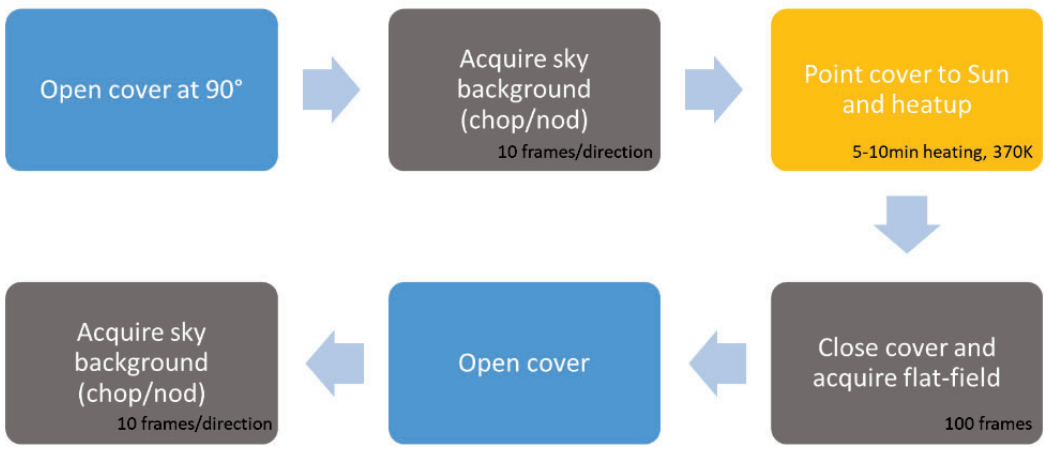

Figure 2: sequence foreseen for the infrared gain calibration, the colours indicate the type of action of the sequence: blue for initialization movements of the CubeSat, light-orange for calibration maneuvers and gray for calibration acquisitions. Gray cells contain also additional information on the specific step.
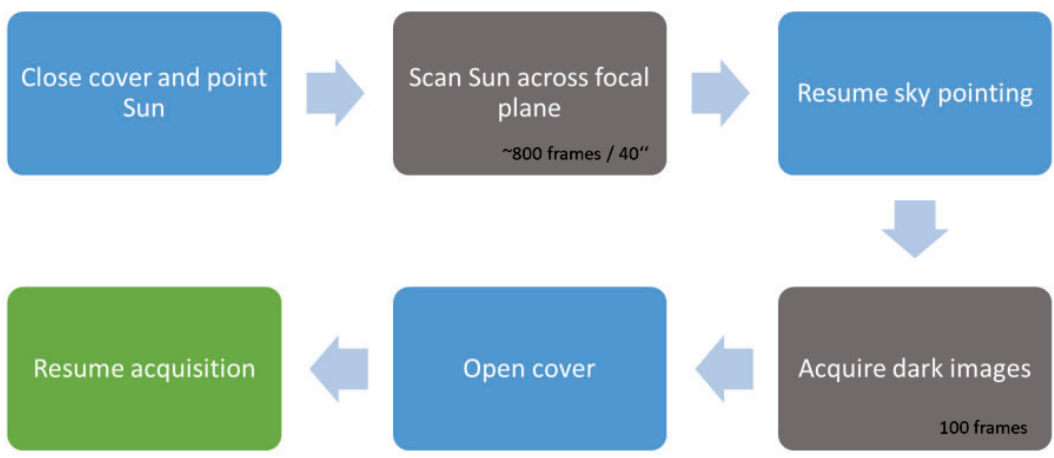

Figure 3: sequence foreseen for the infrared gain calibration, the colours indicate the type of action of the sequence: blue for initialization movements of the CubeSat, green for nominal operation maneuvers and gray for calibration acquisitions. Gray cells contain also additional information on the specific step.

\section{SYSTEM FEASIBILITY}

A key aspect for the functioning of the satellite is the knowledge of its thermal behavior, as both detector and IR background performance are dependent on it. Basing on data available from missions such as [11] and analyses of 6U cubesats available in literature (e.g. [7]), one can expect a certain periodicity in the thermal fluctuations of the structure and bus of about 100min for a LEO orbit. We assume operating temperatures to be centered around 270-275 K, and 
fluctuate with a magnitude of about $30 \mathrm{~K}$ in the case of a thermally passive satellite, with no temperature-controlling elements in place.

With these considerations, it is reasonable to believe that adding a small MLI-type insulation around the structure, as wells as implementing passive thermal control elements for the detectors, would allow for a temperature stability on the order of $5-10 \mathrm{~K}$ for the optical elements and detectors inside the CubeSat. In this configuration, mirrors and detectors respond slower to temperature variations of the external environment, owning also to an efficient thermal decoupling from the structure. Deployable solar panels on four main sides (length) and MLI protection underneath allows the power needed for continuous acquisition as well as insulation. The thermal environment considered in the study is summarized in Table 4.

In addition, to guarantee that the sequences described in section 2.2 can function, a mechanism such as a stepper motor is required to allow for up to 600 calibration cycles to be performed $(1 \mathrm{cycle}=1 \mathrm{open} / \mathrm{close}$ movement $)$. It is assumed that a mechanism of the size required to fit a CubeSat unit, equipped with a dedicated heater allows for such operations to be regularly carried out.

Table 4: main temperature parameters considered in the study.

\begin{tabular}{|l|l|}
\hline Detectors mean operating temperature & $290 \pm 5 \mathrm{~K}$ \\
\hline Internal temperature (optics, mounts) & $\begin{array}{l}280 \pm 10 \mathrm{~K}(\mathrm{M} 2) \\
280 \pm 5 \mathrm{~K}(\mathrm{M} 1, \mathrm{M} 3 \text {, dichroic) }\end{array}$ \\
\hline Calibration cooling rate (max) & $0.1 \mathrm{~K} / \mathrm{s}$ \\
\hline Background temperature variation & $0.02 \mathrm{~K} / \mathrm{s}$ \\
\hline Cover calibration temperature (sun heatup) & $370 \mathrm{~K}$ \\
\hline
\end{tabular}

\subsection{Cover heatup}

Basing on the calibration scenarios of section 2.2, the heatup of the cover can be analyzed by considering two extreme cases, defined by the minimum and maximum solar radiance $\left(1322 / 1414 \mathrm{~W} / \mathrm{m}^{2}\right)$, as well as albedo $(24 \% / 40 \%)$ to which the satellite is exposed to. By considering these boundary conditions at an orbit of $700 \mathrm{~km}$, one can calculate that at equilibrium, the black side of the cover reaches $\sim 270 \mathrm{~K}$.

In this scenario, when exposing a small $8 \mathrm{~cm}$ in diameter-wide cover to the sun, one can estimate that its surface temperature would heat up to $370 \mathrm{~K}\left(\sim 100{ }^{\circ} \mathrm{C}\right)$ in a period of 5-10 minutes. With a fixed duration, yearly differences in the effective surface temperature reachable with a fixed heatup time can be monitored using calibrated temperature sensors (four foreseen) embedded within the cover structure. Preliminary simulations show that such a device exhibits a maximum temperature gradient of $3 \mathrm{~K}$ across its surface after being heated up and closed to irradiate towards the inside of the CubeSat.

\subsection{Sun imaging}

To calibrate the visible channel, pinholes are used to create a blurred image of the sun onto the focal plane. Their dimension should be sized so that enough power is transmitted without saturating, while allowing for a sufficiently high well fill (ideally around 50\%). With the CubeSat configuration under study, one can see that the solar radiance integrated over the detector band $\left(\sim 205 \mathrm{~W} / \mathrm{m}^{2}\right)$, transmitted through the four, $500 \mu \mathrm{m}$ pinholes, produces a well fill of around $60 \%$ in $0.15 \mathrm{~ms}$ (with the CCD parameters of Table 2).

\section{COVER PERFORMANCE}

To allow for an automated observation of the sky, we consider a two-point calibration, based on the acquisition of two different illumination levels (bright flat-fields and dark backgrounds). Which can be written as a function of the instrument gain $g$ and offset $o$ as: 


$$
S_{o b j}=g\left(L_{o b j}\right)+o
$$

Where $S_{o b j}$ is the signal from a stellar object of intrinsic radiance $L_{o b j}$ that is measured by the detector.

By inverting this relation, assuming that the calibration scenes of radiance $L_{1}$ and $L_{2}$ produce signals $S_{1}$ and $S_{2}$ and that the response is adjusted for non-linearity, one can write:

$$
L_{o b j}=K L_{1}+(1-K) L_{2}+\Delta \mathrm{o}, \quad K=\frac{S_{o b j}-S_{2}}{S_{1}-S_{2}}
$$

Where the term $\Delta o$ represents the residual radiance not compensated during the calibration, coming from any changes inbetween calibrations or not accounted by the calibration procedure.

To determine the radiometric stability, we perform the error propagation of the above equation and assume that all uncertainties in the calibration are at first order uncorrelated. Therefore, assuming that constant offsets present in the detection chain are characterized with high precision on ground, and that the NL is known with high precision, one can generically write the radiometric stability as a function of the signal as:

$$
\sigma_{r s}=\sqrt{\left(\sigma_{1} K\right)^{2}+\left(\sigma_{2}(1-K)\right)^{2}+\sigma_{M T}^{2}}+\sigma_{L T}
$$

Where also mid-term effects occurring in-between calibration $\left(\sigma_{M T}^{2}\right)$, as well as long-term effects $\left(\sigma_{L T}\right)$ like a change in the non-linearity are considered. It is anyway assumed that the optical design is chosen to minimize such additional contributors, through straylight control and passive thermal control.

\subsection{Radiometric stability, visible channel}

The contributors to the radiometric instability are differentiated between those present during calibration and those inbetween (mid-term), based on the calibration concept of building a flat-field by observing the sun through the pinholes, and acquiring dark images with the cover closed while pointing to a $\sim$ dark patch of the sky.

The main contributors present during calibration are the following:

- Solar variations: after accounting for the correction of TSI using ephemeris data, instabilities due to its shorterterm cycles [12] are still present.

- Straylight: we assume that a simple light labyrinth is implemented, which reduces the radiation coming through by $10^{-4}$, and that the additional light transmitted through is generated mainly from sun and earth reflections.

- Pointing: the imprecise pointing generates some local variations in the integrated radiance that generates difference when stacking the scanned frames. Assuming a $0.1^{\circ}$ pointing error, this amounts to about $1 \%$ of the total solar radiance transmitted through the pinholes.

- Dark signal: temperature fluctuations in the detection chain causes a small but non-negligible drift in the acquired signal, which is monitored using the unexposed parts of the detector.

- $\quad$ Averaging: residual from averaging of the scenes, based on the number of frames acquired.

In between calibrations and on the long term the following contributors are taken into account:

- Readout noise: white noise of the detector, small effect in magnitude with respect to the total detector dark that is corrected via the unexposed pixels.

- Overall radiometric stability of the detection chain: linked to the temperature stability of the system and readout electronics. We consider this to be equal to a fraction of the measured signal of $2 \%$ at low light levels and improves (decreases) up to $0.5 \%$ for bright signals.

- Drift of the detector linearity over time, which is not recalibrated for, but quantified as being $5 \%$ of the measured signal. 


\section{Scanning of Sun's image}

To analyse the principal effects of scanning the sun's image over the detector, we implement a model of a simple imaging system, where the sun's image is convolved with the PSF generated by a 4-aperture pupil mask. Due to the broadband nature of the detection system, no interference pattern is expected to form.

Firstly, we create a 1D model of the solar disk with a limb-darkened profile, to which we include potential sunspots and flares in the form of narrow peaks across the profile, of varying intensity and width. The profile is then convolved with a simplified 1D PSF of the pupil, created by assuming that the pinholes are distributed symmetrically across $80 \%$ of its diameter, the resulting focal plane image is shown in Figure 4 - left.

Now, if one assumes that sun image is moved across the detector with a speed of $0.6 \%$, it follows that with a frame duration of $0.15 \mathrm{~ms}$ its image is averaged across $\sim 20 \mathrm{px}$ during one exposure. This results in a further blurring of the sun profile that, after stacking all frames together, leads to an overall flat-field such as that one shown in Figure 4 (right), which presents a peak-to-valley non-uniformity of roughly $20 \%$, which we assume can be accurately fit with a polynomial down to $1 \%$ error. Since this is characterizable with high precision, we assume it does not enter the correction as its shape is constant with calibrations (apart from the pointing errors already included).
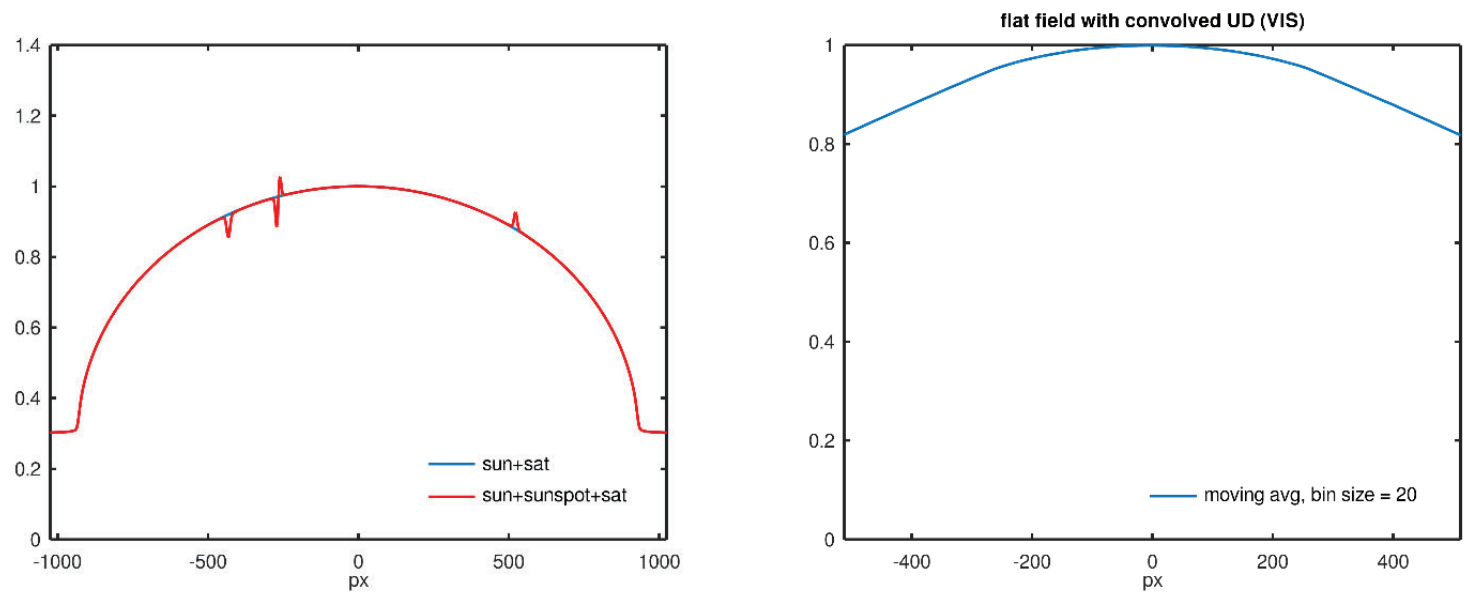

Figure 4: (left) example of the 1D profile of the sun as observed through the closed cover, the blue line represents the ideal limb-darkened only normalized profile, while the red one includes sunspots at selected locations, blurred in intensity and edge sharpness from the convolution with the cover+optics PSF. (right) normalized flat-field profile resulting from stacking the moving image of the sun over the focal plane.

\section{Expected radiometric stability}

Overall, by considering the calibration and mid-term contributors listed above, one can summarize the main contributors to radiometric instability to those of Table 5, which for the detector configuration listed in section 2 leads to a stability ranging between $0.01 \%$ at low signals and $\sim 10^{-5}$ at high signals, as shown in Figure 5. 

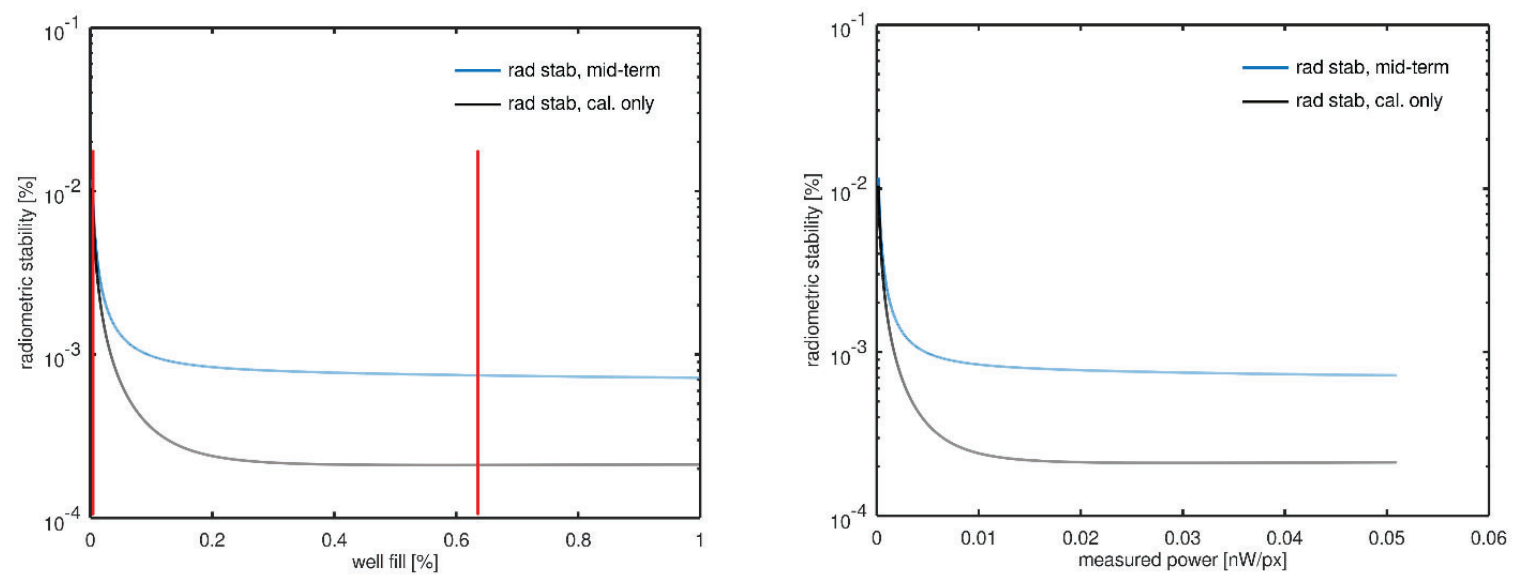

Figure 5: expected radiometric stability of the signal measured in the visible channel, after gain and offset calibration. Left: stability with respect to the detector well fill, with highlighted in red the signal levels expected with dark and sun imaging. Right: same plot, compared with the input power per pixel.

Table 5: list of principal contributors to the radiometric stability of the VIS channel, after calibration.

\begin{tabular}{|l|l|}
\hline Offset error (calibration) & $4 \mathrm{e}^{-}$ \\
\hline Dark signal & $50 \mathrm{e}^{-}$ \\
\hline Straylight & $47 \mathrm{e}^{-}$ \\
\hline Sun 54d variation & $8 \mathrm{e}^{-}$ \\
\hline Sun $\sim$ monthly variation & $5 \mathrm{e}^{-}$ \\
\hline Sun scintillation & $32 \mathrm{e}^{-}$ \\
\hline Pointing error & $142 \mathrm{e}^{-}$ \\
\hline Averaging error (flat-field) & $12 \mathrm{e}^{-}$ \\
\hline Averaging error (dark) & $1 \mathrm{e}^{-}$ \\
\hline Mid-term ageing & $2 \mathrm{e}^{-}$ \\
\hline Offset instability mid-term & $20 \mathrm{e}^{-}$ \\
\hline
\end{tabular}

\subsection{Radiometric stability, mid-IR channel}

Similarly to the visible channel, contributors to the radiometric instability are differentiated between those present during calibration and those in-between calibration events. In this case, the flat-field is measured by observing the hot cover, while the background is corrected for using a series of chop/nod acquisitions of the dark sky. The main contributors are categorized below, it is considered that for the duration of the calibration the detector temperature remains sufficiently stable not to play a significant role in the calibration instabilities - considering also that it is monitored anyway using the parts of the detector unexposed to radiation.

The main contributors present during calibration are the following:

- Error in determining the cover true temperature: based on measuring the temperature with 4 calibrated sensors, each having a resolution of $50 \mathrm{mK}$ and an absolute accuracy of $1 \mathrm{~K}$. 
- $\quad$ Radiance error due to the cooling of the cover while acquiring calibration images (Table 4).

- Instability of the background reflected back into the optical system due to the non-perfect emissivity of the cover.

- $\quad$ Averaging: residual from averaging of the scenes, based on the number of frames acquired.

- Straylight: we assume that a simple light labyrinth is implemented, which reduces the radiation coming through by $10^{-4}$, and that the additional light transmitted through is generated mainly from sun and earth reflections.

- Daily background variations between different chop/nod pairs observed at random positions over subsequent orbits.

In between calibrations and on the long term the following contributors are taken into account:

- $\quad$ Readout noise: white noise of the detector, negligible in magnitude since calibration takes advantage of correction with unmasked pixels.

- Overall radiometric stability of the detection chain: linked to the temperature stability of the system and readout electronics. We consider this to be equal to a fraction of the measured signal of $2 \%$ at low light levels and improves (decreases) up to $0.5 \%$ for bright signals.

- Drift of the detector linearity over time, which is not recalibrated for, but quantified as being 5\% of the measured signal

- Temperature fluctuations of the mirrors: this is the main source of instability between calibrations and is weighted differently between each mirror, assuming the highest fluctuation $(10 \mathrm{~K})$ for the outermost M2 which decreases to $5 \mathrm{~K}$ for the elements placed more inside the structure.

\section{Modelling cover instabilities}

Temperature gradients on the surface of the cover and the limited number of sensors that can be fitted on it, leads to an error in the determination of its true temperature (and consequently radiance). Assuming a maximum peak-to-valley temperature difference of $3 \mathrm{~K}$ after heatup and cover closure, one can estimate the effective temperature error by considering how the sensors are placed over the surface and their measurement accuracy.

To quantify this parameter we follow a statistical (Monte-Carlo) approach, by generating a number of different temperature distributions (temperature nodes) accounting for a certain smoothness in the gradient over the surface (no sharp variations between consecutive nodes). For each distribution, we assume that sensors are placed such that the temperature they measure differs from that one of any neighboring point by no more than a given value $(0.5 \mathrm{~K}$ in our case). The readings from all four sensors are used to determine a mean temperature and from it the mean radiance $\left(\left\langle I_{\text {cal }}\right\rangle\right)$ emitted by the cover. After that, the error is determined from the standard deviation between $\left\langle I_{\text {cal }}\right\rangle$ and the true radiance calculated by considering each temperature node. Finally, the $85 \%$ percentile of all possible distributions determines the error accounted for in the computation. 

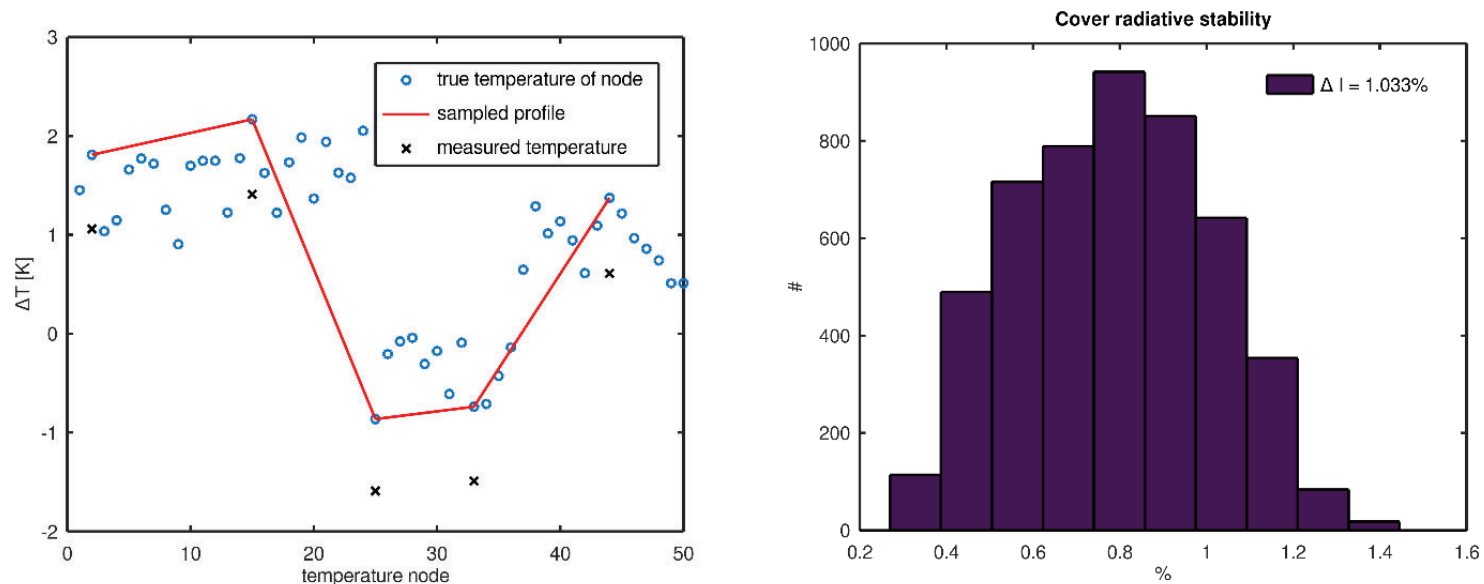

Figure 6: (left) example of a flattened cover temperature profile generated out of a MC run, where each blue dot represents the temperature of a point (node) in the surface of the cover, the red line the simplified profile around which each temperature node is defined and the black crosses the values measured by each sensor, all with respect to the mean calibration temperature of $370 \mathrm{~K}$. (right) histogram of the radiance error in $\%$ of the calibration radiance obtained from all $\mathrm{MC}$ runs with highlighted the $85 \%$ percentile value on the top-right.

In addition to the uncertainty in determining the cover's true temperature, we assume that over time the emissivity of the cover degrades unequally due the uneven exposure to ageing events (e.g. radiation), thus reducing further the ability of scaling for the correct calibration radiances. In this scenario, we consider that a given area of the cover can degrade its emissivity by maximum $5 \%$ over the course of the mission, and that patches large up to $10 \%$ of the pupil diameter can degrade evenly at the same time. Also in this case a Monte-Carlo approach is well-suited for the analysis, allowing one to generate several degraded intensity profiles of the cover to determine the residual variation between the BOL radiance slope (assumed to be without local degradations) and a degraded (EOL) one. The $85 \%$ percentile of all residuals determine the radiometric error to be included when computing the overall radiometric stability.

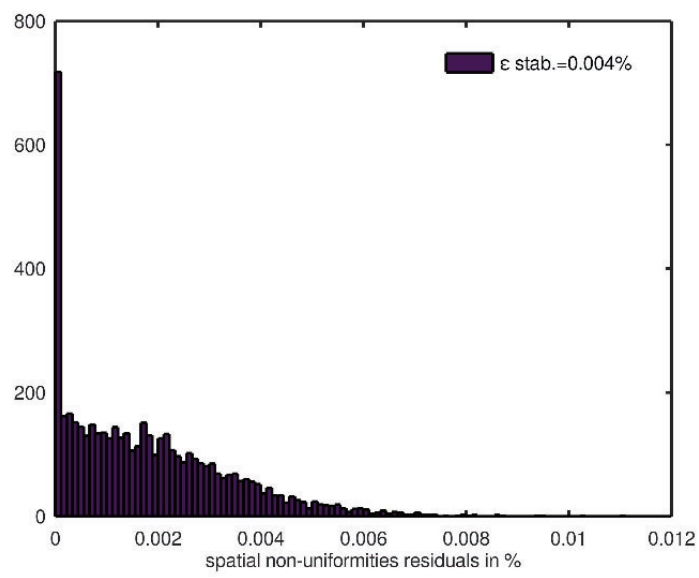

Figure 7: result of Monte-Carlo analysis of the global emissivity degradation across the cover. The values are expressed in $\%$ of the effective emissivity taken as reference from the BOL value.

\section{Modelling IR background calibration}

As the CubeSat circles around Earth, its temperature will change principally because of the different exposure to the sun and Earth's albedo, causing the infrared background to fluctuate with it. This combines with the variation in intensity of 
the diffuse IR background, as a function of the sky location being observed. A regular and continuous background calibration allows to automatically follow the combination of these two effects, reducing the radiometric instability to the local differences between subsequent chop/nod pairs, with a cycling duration of a bit more than one orbit (thermal cycles timescales). Based on these assumptions, we consider the IR background to be weighted with the changing radiation levels present every chop/nod acquisition, due to the different pointing in the sky exposed to different levels of zodiacal light flux ([13] [14]). We simulate the combined effect by considering that a weighted "deep space" temperature cools down the CubeSat differently, depending on the position over the orbit. Every acquisition, its magnitude is calculated by assigning a different intensity to the diffuse IR background based on the probability of pointing to different patches of the sky (higher if observing near the ecliptic, lower when perpendicular to it).

In particular, during every chop/nod sequence, the background will vary as the cubesat points at different areas of the sky, thus also cooling/heating the optics differently with respect to the nominal observation mode. Overall, this follows the temperature fluctuations of the cubesat and results in a small change between the background used for calibration and the effective one (of the true scene). In the simulation we consider that the temperature cyclically change around its reference point by $\pm 10 \mathrm{~K}$ with a temperature change rate of maximum $0.02 \mathrm{~K} / \mathrm{s}$. Then we run through a Monte-Carlo analysis to determine the effect of daily acquiring 100 different chop/nod background for different drifting temperature profiles. Finally, the radiometric stability of the background correction is weighted between the moving average of each group of acquisitions and the oscillation of the nominal background while observing (Figure 8).

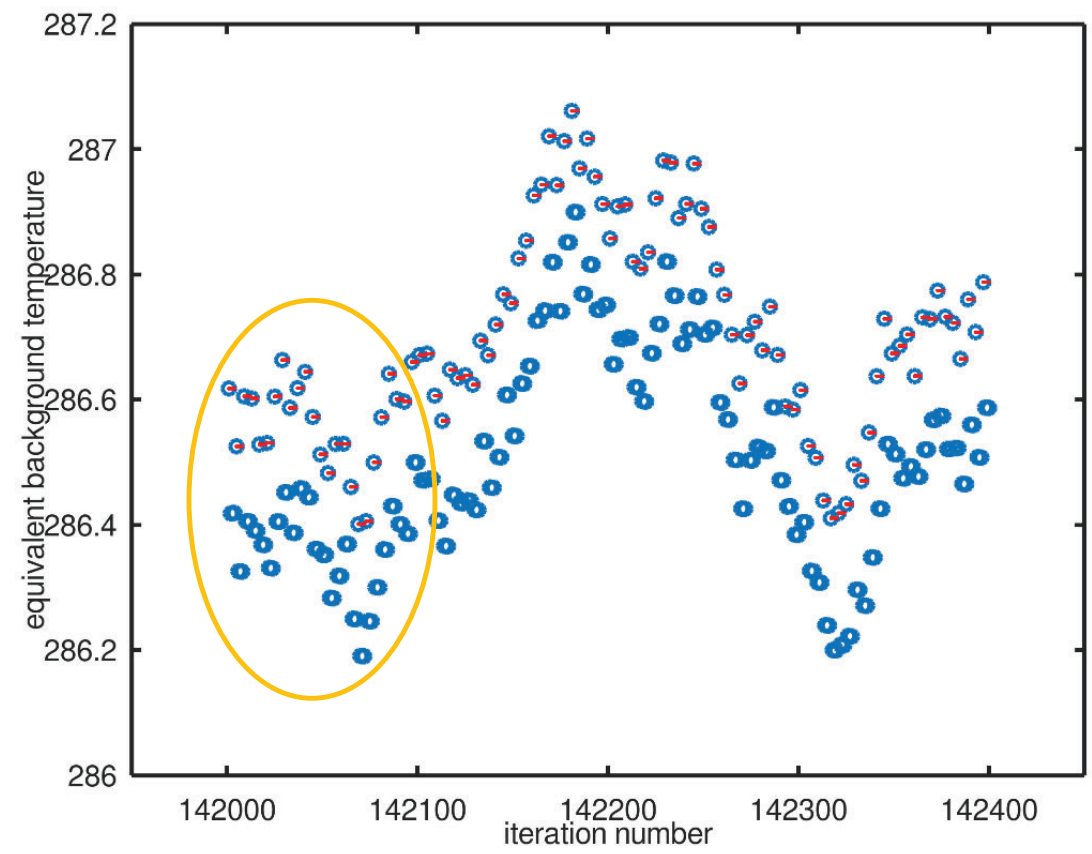

Figure 8: extract of the MC simulation showing a group of consecutive IR background calibrations, measured at different levels of illumination coming from the zodiacal light. The values are expressed in terms of equivalent blackbody radiance in Kelvin. The blue circles represent the effective calibration temperature as the system's temperature changes depending on the levels of "deep space" exposure. The red lines instead indicate the CubeSat's temperature at the start and end of an acquisition sequence, which can be identified by the yellow ellipse. In total four consecutive calibrations are shown in the graph.

\section{Expected radiometric stability}

Overall, by considering the calibration and mid-term effects listed above, one can summarize the main contributors to radiometric instability to those of Table 6 , which for the detector configuration listed in section 2 leads to a stability ranging between $0.2 \%$ for low signals and $0.01 \%$ for high signals, as shown in Figure 9 . The main reason for the large instability 
at low signals lies in the background fluctuation of the optical elements between daily calibrations - in fact if one accounts only for the calibration errors the instability reaches a minimum of $0.005 \%$ for signals close to the average level of the IR background ( $10 \mathrm{NEP})$.

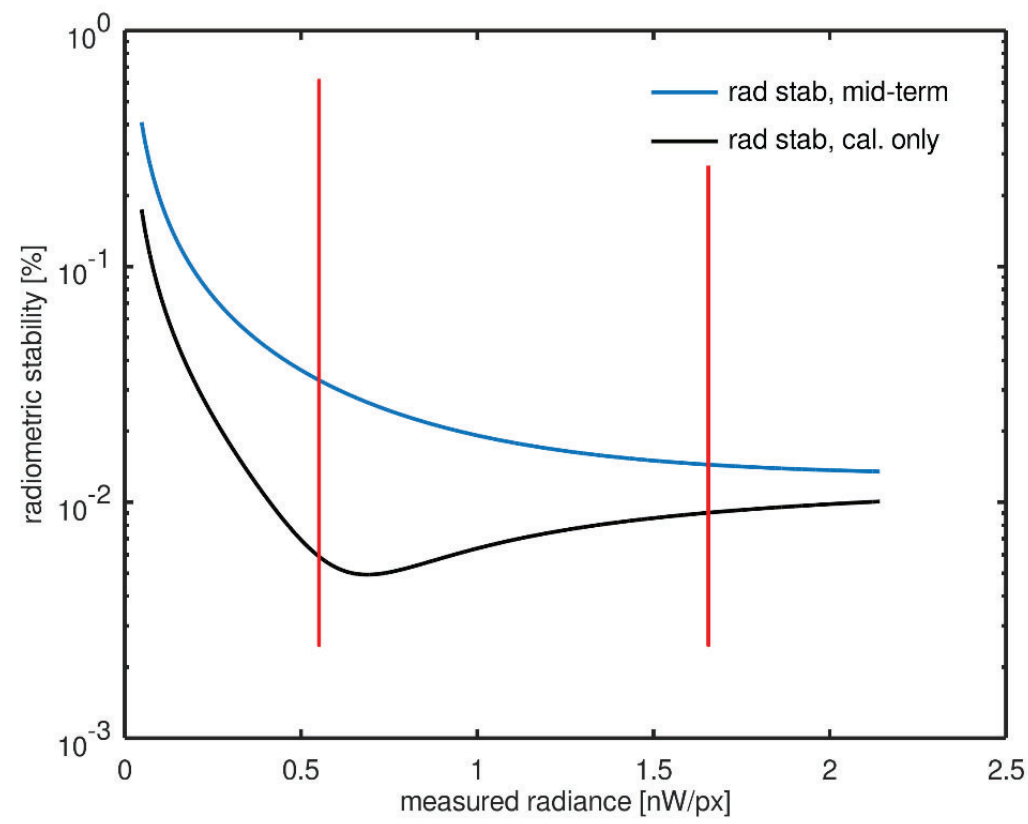

Figure 9: expected radiometric stability of the signal measured in the infrared channel, after gain and offset calibration. The stability with respect to the input power per pixel is shown, with highlighted in red the signal levels expected during background and hot cover imaging.

Table 6 list of principal contributors to the radiometric stability of the mid-IR channel, after calibration. All converted in radiance units.

\begin{tabular}{|l|l|}
\hline Background instability (chop/nod) & $0.06 \mathrm{pW} / \mathrm{px}$ \\
\hline Flat-field SNR & 300 \\
\hline Background SNR & 100 \\
\hline M1 stability & $4.53 \mathrm{pW} / \mathrm{px}$ \\
\hline M2 stability & $9.54 \mathrm{pW} / \mathrm{px}$ \\
\hline M3 stability & $5.02 \mathrm{pW} / \mathrm{px}$ \\
\hline Straylight & $0.08 \mathrm{nW} / \mathrm{px}$ \\
\hline Cover effective temperature error & $0.02 \mathrm{nW} / \mathrm{px}$ \\
\hline Cover cooling error & $0.40 \mathrm{nW} / \mathrm{px}$ \\
\hline Background instability during calibration & $0.02 \mathrm{nW} / \mathrm{px}$ \\
\hline Detector NEP & $0.06 \mathrm{nW} / \mathrm{px}$ \\
\hline
\end{tabular}




\section{CONCLUSIONS}

We analysed the radiometric performance of a potential $6 \mathrm{U}$ CubeSat design to be employed for automatic photometric surveys of the sky at VIS and mid-IR wavelengths. We demonstrated that by employing an externally-mounted and moveable cover with an IR-black coated surface and small pinholes, solar radiation can effectively be used to perform a regular radiometric calibration automatically.

Overall, the calibration strategy discussed in the paper allows one to reach a photometric repeatability in the order of $0.01 \%$ or better in the visible range $(400-900 \mathrm{~nm})$ and $0.2-0.01 \%$ in the IR around $10 \mu \mathrm{m}$.

This study is to be considered as a proof of concept that can be adapted to the needs of specific science cases, potentially allowing for higher-performing systems than that one considered here, which is mainly based on reference figures and technical solutions publicly available in literature.

\section{REFERENCES}

[1] Raskin, G. et al., "CUBESPEC: Low-cost space-based astronomical spectroscopy," Proceedings of SPIE Astronomical Telescopes + Instrumentation, (2018).

[2] Schifano, L. et al., "Development of a space-based nulling interferometer to detect and characterize exoplanets," Proceedings of International Conference of Space Optics (ICSO), 104 (2018).

[3] Weiss, W. et al., "BRITE-Constellation: Nanosatellites for Precision Photometry of Bright Stars," Publications of the Astronomical Society of the Pacific, 126 (940), 573-585 (2014).

[4] Allthorpe-Mullis, E. et al., "CubeSat Camera: A Low Cost Imaging System for CubeSat Platforms," 7th Interplanetary CubeSat Workshop, 1-9 (2018).

[5] Vieira, H. et al., "Development of a low cost space telescope for Earth remote sensing from a 12U CubeSat," Proceedings of International Conference of Space Optics (ICSO), 11180 p.25 (2018).

[6] Tsitas, S. R. et al., "6U CubeSat design for Earth observation with $6.5 \mathrm{~m}$ GSD, five spectral bands and 14Mbps downlink," The Aeronautical Journal 114 (1161), 689-697 (2010).

[7] Jin, H. et al., "Optical Design of a Reflecting Telescope for CubeSat," Journal of the Optical Society of Korea, 17 (6), 533-537 (2013).

[8] Muslimov, E. R. et al., "Optical Design for a CubeSat: Unobscured Telescope, Using Freeform Mirrors and a Curved Detector," Russian Aeronautics, 61 (1), 1-7 2018.

[9] Clark, M., "Imaging Systems for CubeSats," The Planetary CubeSats Symposium, (2018).

[10] Almasri, M. et al., "Self-supporting uncooled infrared microbolometers with low-thermal mass," Journal of Microelectromecanical Systems, 10 (3), 469-476 (2001).

[11] Rossi, S. et al., "Thermal model for cubesat: A simple and easy model from the Swisscube's thermal flight data," Proceedings of the International Astronautical Congress 13, 9919-9928 (2013).

[12] Lee, J. N. et al., "The 27-day rotational variations in total solar irradiance observations: From SORCE/TIM, ACRIMSAT/ACRIM III, and SOHO/VIRGO," Journal of Atmospheric and Solar-Terrestrial Physics 132, 64-73 (2015).

[13]Leinert, C. et al., "Mid-infrared spectrum of the zodiacal light observed with ISOPHOT," Astronomy \& Astrophysics, 393 (3), 1073-1079 (2002).

[14] Ootsubo, T. et al., "IRTS observation of the mid-infrared spectrum of the zodiacal emission," Earth, Planets and Space, 50 (6-7), 507-511 (1998). 